\title{
Cardiovascular Risk with Non-steroidal Anti-inflammatory Drugs: Clinical Implications
}

\author{
Alfonso E. Bello $\cdot$ Robert J. Holt
}

Published online: 31 July 2014

(c) The Author(s) 2014. This article is published with open access at Springerlink.com

\begin{abstract}
In February 2014, the US Food and Drug Administration (FDA) convened an advisory committee meeting to discuss the accumulated data relating to the cardiovascular risk of non-steroidal anti-inflammatory drugs (NSAIDs) and the potential implications on the class prescription labeling. The committee recommended, though not unanimously, that (1) the current data does not support the conclusion that naproxen has a lower risk of thrombotic events than other NSAIDs; (2) there is no latency period for the risk of cardiovascular thrombotic events; (3) there are some patient populations at increased risk for events; and (4) equipoise remains in the major ongoing trial designed to address these issues further. The clinical implications of the FDA deliberations as well as the recently published meta-analyses and observational studies are discussed. With the information available today, there is insufficient evidence to conclude that there are significant differences between the approved NSAIDs with regard to the potential for cardiovascular events. An approach for balancing the major risks associated with NSAIDs is suggested. Clinicians should continue to use the current FDA NSAID labeling language to guide their decision making for individual patients until such time as the FDA makes changes.
\end{abstract}

\footnotetext{
A. E. Bello ( $\square)$

College of Medicine, University of Illinois-Chicago, Illinois

Bone and Joint Institute, LLC, 2401 Ravine Way, Glenview,

IL, USA

e-mail: abello@ibji.com

R. J. Holt

College of Pharmacy, University of Illinois-Chicago,

1721 North Woods Way, Vernon Hills, IL, USA

e-mail: rholt5@uic.edu
}

\section{Key Points}

New data from observational studies and metaanalyses of randomized controlled trials have suggested that naproxen may be associated with a lower risk of cardiovascular thrombotic events as compared to other NSAIDs.

An FDA advisory committee was convened to review the new data, and a majority of the panel did not find the data sufficient to suggest major prescription labeling changes and suggested that PRECISION, the major trial designed to address these issues, continue unchanged.

\section{Introduction}

Prescription non-steroidal anti-inflammatory drug (NSAID) use is prevalent in the USA, reflecting the aging of the population and the concomitant rise in musculoskeletal diseases, particularly osteoarthritis (OA) and rheumatoid arthritis (RA). From October 2011 to September 2012, the top five NSAIDs accounted for nearly 86 million dispensed prescriptions for 44 million unique patients [1]. This level of use and acceptance reflects the well-recognized anti-inflammatory and analgesic properties of these agents. Historically, research had identified these positive effects to be associated with inhibition of cyclooxygenase (COX)-2-mediated inflammation as opposed to $\mathrm{COX}-1$, which is responsible for constitutive prostaglandin synthesis that, among other things, protects the 
gastrointestinal (GI) track and affects platelet homeostasis. As the prototypic NSAIDs block both COX-1 and COX-2 to varying degrees, both positive (anti-inflammatory/ analgesia) as well as negative (bleeding/GI complications) effects could be expected. It was intuitive to pursue research on this COX-2 hypothesis (preferential COX-2 inhibition while sparing COX-1 inhibition) to determine if more effective or safer ways to balance the risks with the benefits from NSAIDs could be uncovered.

Research began in the 1990s to better understand COX2 selectivity and culminated in the introduction of COX-2 selective inhibitors (coxibs), along with the hope that we could push doses higher for greater anti-inflammatory effect while reducing GI side effects and improving tolerability. Though not powered to show a difference in efficacy, the large pivotal trials for the first two coxibs available in the USA showed non-inferiority to the traditional non-selective NSAIDs (nsNSAIDs), ibuprofen, diclofenac, and naproxen, in the treatment of OA [2-4], reinforcing the general observation that at equivalent doses all NSAIDs are equally efficacious. Coxib studies, however, demonstrated a reduction in the risk of NSAIDinduced adverse GI effects (particularly GI ulcers) compared with nsNSAIDs, but pharmaceutical sponsors were never able to provide compelling enough evidence to the Food and Drug Administration (FDA) for formal labelindicated approval of a reduction in GI complications [57]. This lack of regulatory recognition of the benefits of coxibs nevertheless did not temper their early acceptance in the clinical community.

\section{Cardiovascular (CV) Adverse Events and the "COX- 2 Hypothesis"}

In 2001, a study published in the New England Journal of Medicine on the GI comparative safety of rofecoxib (the second coxib approved in the USA) reported a myocardial infarction (MI) rate significantly higher than that with naproxen (0.4 vs. $0.1 \%)$ [7]. Interestingly, the authors concluded that the difference was likely due to a favorable secondary cardiovascular (CV) protective effect of naproxen on platelet function since the effect seemed to occur in those who were at risk for $\mathrm{CV}$ events (i.e., were candidates for cardioprotective doses of aspirin, but were not receiving it). In the group that was not at risk for $\mathrm{CV}$ events, there was no significant difference in the incidence of MI between those who received naproxen or rofecoxib. Additional data from this trial, which were released later, indicated that rofecoxib did indeed carry an independent thrombotic risk, especially as the dose increased $[8,9]$, and that any benefit from the prevention of one complicated upper GI event came at the cost of one serious thrombotic event [9]. Rofecoxib was voluntarily removed from the market in 2004 after the results of a placebo-controlled trial found almost a doubling of serious CV events [10] and that the risk was elevated up to a year after stopping the drug [11]. Regulatory actions shortly occurred on other coxibs; valdecoxib was removed from the market, and its prodrug, parecoxib, received a non-approvable letter from the FDA in 2005. Celecoxib remains the only coxib available on the US market [1].

Many of the CV safety issues that this early foray into COX-2-specific inhibition uncovered are still open, and concerns have expanded to the NSAID class at large. Among the nsNSAIDs, small differences in CV safety have been observed, and the relative effects of the agents in this class have been debated. The relative CV safety among the class will remain a central question for clinicians and regulators for the next decade.

\section{FDA Advisory Meetings and History of Non-steroidal Anti-inflammatory Drug (NSAID) Class Labeling}

In 2005, the FDA held an advisory committee meeting to discuss the data that had emerged from the coxib trials, including data on drugs under development. Largely on the basis of the trials discussed above with rofecoxib and those of other drugs under development (etoricoxib, lumiracoxib), the committee determined that all NSAIDs confer an increased risk for serious and potentially life-threatening CV events (defined as thrombotic events, MI, and stroke) and that the data do not allow a rank ordering of risk potential [1].

On the basis of the discussion and recommendations from the committee at that time, the FDA took the following key actions on all prescription NSAID labels, adding (1) a "black box" warning highlighting the potential for increased risk for CV events and serious lifethreatening GI bleeding, ulceration, and perforation; (2) statements indicating patients with, or at risk for, CV disease and the elderly may be at greater risk, and that these reactions may increase with duration of use; (3) a contraindication for use after coronary artery bypass graft surgery on the basis of reports with valdecoxib/parecoxib; (4) language that the lowest dose should be used for the shortest duration possible; and (5) wording in the warning section that there is no evidence that the concomitant use of aspirin with NSAIDs mitigates the CV risk, but that it does increase the GI risk.

Furthermore, the FDA requested that Pfizer, the company that markets celecoxib, complete a post-marketing trial to examine these issues in more detail, and the Prospective Randomized Evaluation of Celecoxib Integrated Safety versus Ibuprofen Or Naproxen (PRECISION) Trial was started in 2006. The study was designed to examine the 
non-inferiority of celecoxib, naproxen, and ibuprofen on first occurrence of a composite $\mathrm{CV}$ primary endpoint $(\mathrm{CV}$ mortality, non-fatal MI, non-fatal stroke) among randomized OA or RA patients with, or at high risk for, $\mathrm{CV}$ disease expected to need high-dose NSAID therapy [12]. Low-dose aspirin and gastroprotective therapy with esomeprazole are allowed as needed, and GI outcomes are to be monitored prospectively. Patient enrollment had approached $>95 \%$ $(22,621)$ of target $(23,750)$ as of February 2014 , but the event rate has been lower than expected and the power of the study was reduced from 90 to $80 \%$, lowering the events needed from 762 to 580 and extending the end date to December 2015, 2 years later than originally planned [1].

In February 2014, the FDA convened another advisory committee meeting based on recently published metaanalyses and observational studies calling into question some of the previous assumptions guiding the US NSAID class labeling. In 2011, an observational study from the Danish National Registry was published that indicated that patients who had previously had an MI were at risk for recurrent MI and death as early as 1 week after starting NSAID therapy [13]. The authors concluded their report by saying "neither short- nor long-term treatment with NSAIDs is advised in this population." Furthermore, a metaanalysis of randomized NSAID trials by the Coxib and traditional NSAID Trialists' (CNT) Collaboration published in the Lancet in May 2013 concluded that "the vascular risk of high-dose diclofenac, and possibly ibuprofen, are comparable to coxibs, whereas high-dose naproxen is associated with less vascular risk than other NSAIDs. Although NSAIDs increase vascular and gastrointestinal risks, the size of these risks can be predicted, which could help guide clinical decision making" [14]. Shortly after publication of this data, the European Medicines Agency (EMA) revised the labeling of diclofenac to be in line with that of coxibs, which have stronger prescribing warnings than nsNSAIDs in Europe [15].

Pre-meeting materials for the 2014 advisory committee meeting included FDA staff review of the published data, including one from a Medical Officer in the Office for Pharmacovigilance and Epidemiology, who concluded the following [1]: (1) there appears to be less thrombotic CV risk with naproxen and a higher risk with diclofenac as compared with other nsNSAIDs; (2) CV risk is observable from the beginning of NSAID therapy; (3) the relative risk (RR) for NSAID-induced CV events is the same in healthy individuals as in those at risk for $\mathrm{CV}$ events, but the absolute risk is higher for those patients at risk for CV events; (4) observational studies in aggregate indicate that both nsNSAIDs and coxibs are associated with stroke, albeit at a lower rate than MI, and there is no pattern of relative hazard among the NSAIDs; (5) some evidence exists that aspirin can decrease $\mathrm{CV}$ risk associated with
NSAIDs, but naproxen and ibuprofen (the most studied NSAIDs) can inhibit the cardioprotection of aspirin when administered in close time proximity; (6) there is some evidence that naproxen may have a lower $\mathrm{CV}$ risk than ibuprofen, but ibuprofen has lower GI risk than naproxen; and (7) equipoise no longer exists in the PRECISION trial and it should be stopped.

\section{FDA Advisory Committee Discussion and Vote on CV Issues}

At the February 2014 meeting, background presentations were presented by authors of the meta-analyses and observational studies, FDA officials, industry representatives, as well as guest speakers, including the lead investigator for the PRECISION trial. The following topics were specifically discussed [1]:

4.1 Does the Accumulated Data Support a Clinically Significant Difference in Risk for CV Thrombotic Events for Any of the NSAIDs?

A number of panelists agreed that the data appear to support a differential risk with NSAIDs, although differing study characteristics (unequal doses, acute/chronic use, low/high CV risk, and aspirin use or not) complicate the interpretation of the CNT meta-analysis. Most agreed that the data were suggestive of a differential benefit with naproxen but were not strong enough to support a label claim, and the indirect NSAID comparison approach of the CNT meta-analysis made some uncomfortable, especially given that most of the confidence intervals overlapped. The FDA addressed questions from the committee on the appropriateness of using meta-analytic and observational data to make changes in the prescribing labels. FDA staffers echoed comments previously published in the debriefing materials, that the use of observational and meta-analytic studies informing regulatory decisions previously "were focused on evaluating efficacy rather than safety. As such, these examples are not directly relevant to a meta-analysis primarily done to assess harms, as in the case of the CNT meta-analysis. The standard for establishing efficacy or clinical benefit based on clinical trials is well established and is not based on meta-analysis. In contrast, best practices for safety related meta-analysis of clinical trials are not well established and the FDA has not issued any Guidance on the subject" [1]. The committee voted 16 to nine that the data do not support naproxen's lower CV thrombotic risk as compared with other NSAIDs. Several panel members indicated they voted no because of concerns that giving preferential CV labeling to naproxen may inadvertently cause harm by increasing use and its 
associated GI risk. Several also pointed out that the option of using it preferentially for appropriate patients exists as a personal clinical decision presently.

\subsection{Does the Weight of Evidence Support \\ Reconsideration of Advice Regarding the Latency of CV Thrombotic Risk?}

The committee voted 14 to 11 that the evidence indicates that there is no time period where patients taking NSAIDs may be considered to not be at CV risk. The majority of the committee indicated that there is risk across time and the labeling should reflect this. Those that disagreed indicated that not all studies demonstrate this and many that do are with pre-existing CV disease or at risk patients, on high doses, and/or without low-dose aspirin (especially the coxib studies).

4.3 Based on the Available Data, Is It Appropriate to Consider Any Restrictions or Specific Warnings for Those Populations Who Are at Higher Absolute Risk for CV Thrombotic Events with NSAID Use? Potential Options Include but Are Not Limited to Extending the Contraindication in Certain Subpopulations [e.g., Patients Immediately PostMyocardial Infarction (MI)] or Including a Statement in the Boxed Warning Regarding the Increased Absolute CV Thrombotic Risk in the Post-MI or Heart Failure Populations

Most of the panel indicated that they agreed with the notion of calling out patients at risk, but many were reluctant to make specific population recommendations, with the exception of post-MI and RA patients, who may be at higher risk. In a large cohort study, even short-term treatment with NSAIDs has been found to increase the risk for CV death and recurrent MI in the post-MI patient population, and, therefore, these agents should be avoided if possible in this population [13]. In regard to heart failure, the committee mostly supported specific labeling, on the basis of data from the CNT meta-analysis, which indicated that all NSAIDs predispose to heart failure, but many were reluctant to suggest a contraindication given the benefit in appropriate patients. They agreed that those with coronary artery disease are at risk and the prescription labeling should be strengthened in this regard. A vote was not taken on this issue.

\subsection{Are There Any Changes That Should Be Made to the PRECISION Trial to Respond to the Concerns That Have Been Raised?}

Most panelists voiced support for equipoise between treatment arms in the study, viewed it as an important trial, and felt that it should not be stopped. While some expressed concern about the design and whether it will answer the question on differential CV risk, many suggested it is the best chance to answer the question. Some suggested that if there was evidence of harm, the study would have been stopped by the safety monitoring committee and, therefore, it should continue. A vote was not taken on this issue.

\section{Clinical Implications}

Although the FDA is yet to announce any changes in NSAID prescribing labeling, they generally follow the recommendations of the advisory committee. Given the recent data and FDA discussions, what clinical conclusions can we make? Among the FDA advisory committee, $36 \%$ of them were convinced that the current evidence justified the conclusion that naproxen had a lower CV thrombotic risk, and a few of them admitted that they would change their prescribing habits on the basis of this data, but the majority were not convinced [1].

One of the most controversial issues with the data is the use of indirect comparisons in the calculation of the CV hazard rate ratios (number of events/number of patient years) in the CNT analysis [14]. Direct comparisons using both trial- and patient-level data were used to calculate these ratios for the coxibs versus placebo and nsNSAIDs. On the contrary, a combination of direct and indirect methods was used to compare individual nsNSAIDs to placebo. The indirect method is acceptable in trials with little heterogeneity [1]. An FDA statistical review found that the CNT meta-analysis generally fit the acceptable standards for such a method, with the exception of 'indication for treatment differences' which exists among the coxib studies (Alzheimer's and colon adenoma cancer prevention) [1]. Importantly, the analysis provides only comparative data of individual nsNSAIDs and coxibs versus placebo and coxibs versus individual NSAIDs.

There are no direct or indirect comparisons among individual nsNSAIDs in the report because of the lack of randomized head-to-head trials, which is a key clinical question. One way to estimate whether two risk ratios are different from one another is the use of a ratio of relative risk (RRR) calculation [16]. Using an RRR calculation on the major vascular event RRs obtained in the CNT metaanalysis for each of the nsNSAIDs versus placebo, one can estimate that the data support the conclusion that diclofenac's risk is higher than naproxen's, but that other comparisons are not significant (Table 1). This is not surprising in that of the three nsNSAIDs studied, diclofenac is the only one shown to have a higher CV risk than placebo in the CNT analysis [14] and a higher CV mortality and 
morbidity in observational studies [17]. It is surprising given these findings that more discussion at the FDA advisory meeting was not centered on diclofenac, as the data would seem to indicate that it has a CV risk similar to that of rofecoxib, and is potentially deserving of stricter labeling than the other nsNSAIDs, as the EMA has done.

Finally, an important issue of competing GI risk is reported in the CNT meta-analysis and emphasizes the need to put this in perspective when we are making clinical decisions. The trials clearly show that coxibs statistically significantly reduce the risk of upper GI complications when compared with ibuprofen, naproxen, and diclofenac, but have higher vascular CV risk as compared with naproxen and placebo [14]. Given that naproxen is thought to have the highest level of upper GI complications, any presumed $\mathrm{CV}$ risk mitigation should be met with the equal understanding of that increased GI risk.

\section{Treatment Implications}

It is very difficult to take population-based data and apply it to individual patients, and as such, all published data

Table 1 Calculated ratios of relative risk (RRR) (95\% confidence interval) for major vascular events with non-selective NSAIDs from the CNT collaboration meta-analysis [14]

\begin{tabular}{ll}
\hline NSAID Comparators & RRR (CI) \\
\hline Ibuprofen vs. naproxen & $1.55(0.88-2.74)$ \\
Ibuprofen vs. diclofenac & $1.02(0.60-1.74)$ \\
Diclofenac vs. naproxen & $1.52(1.03-2.22)$ \\
\hline
\end{tabular}

CI confidence interval, CNT Coxib and traditional NSAID Trialists', $N S A I D s$ non-steroidal anti-inflammatory drugs, $R R R$ ratios of relative risk should be viewed in that light. Patients are very rarely exactly at the mean or in aggregate like those in randomized clinical trials, so we should use the wisdom gained from the clinical data and professional guidelines to help us mold our individual patient decisions. Herein is one approach informed from the current data and previously published expert reviews [18, 19].

One way to analyze the tradeoff of NSAID-induced CV and GI risk is by considering levels of individual patient risk, as presented in Table 2. All patients, regardless of risk factors, are at some GI and CV risk simply by taking the NSAID; therefore, all patients should receive the lowest effective dose for the shortest time period. For patients with increasing GI risk, we know we can mitigate this risk by using NSAIDs known to have lower intrinsic GI risk, such as ibuprofen or celecoxib, and by using concomitant gastroprotective agents such as proton pump inhibitors (PPIs) or histamine $\mathrm{H}_{2}$ receptor antagonists (H2RAs) individually or in fixed-dose combination products [18, 19]. Though NSAIDs do appear to have intrinsic differences in GI risk, this may be lost at higher dosages [14, 19]. Strategies to reduce CV risk with NSAIDs are less well documented, and even the protective benefit of aspirin in NSAID users is still debated. For example, the CNT analysis found that vascular event risk did not differ in aspirin users at baseline versus non-users in those who used coxibs $[\mathrm{RR}=1.33(95 \%$ CI $0.67-2.63)$ vs. $1.40(0.99-1.97)]$ [14]. The increased GI risk with concomitant aspirin and NSAIDs is well established, however [18]. Naproxen might have a more favorable $\mathrm{CV}$ risk profile than coxibs and diclofenac at higher doses, and it seems prudent to consider this when there are no major contraindications for its use, but we do not know if this apparent benefit is obviated or enhanced by aspirin.

Table 2 GI and CV risk assessment and potential NSAID treatment choices

\begin{tabular}{|c|c|c|c|c|}
\hline $\begin{array}{l}\text { Patient } \\
\text { risk level }\end{array}$ & No GI risk & Low GI risk & Moderate GI risk & High GI risk \\
\hline $\begin{array}{l}\text { No } \mathrm{CV} \\
\text { risk }\end{array}$ & $\begin{array}{l}\text { Lowest effective dose of } \\
\text { most effective NSAID }\end{array}$ & $\begin{array}{l}\text { NSAID with least GI } \\
\text { risk (ibuprofen) }\end{array}$ & $\begin{array}{l}\text { 1. NSAID with least GI risk +PPI/ } \\
\text { H2RA (ibuprofen) } \\
\text { 2. Celecoxib }\end{array}$ & $\begin{array}{l}\text { 1.Celecoxib plus PPI/H2RA } \\
\text { 2. Ibuprofen plus PPI/H2RA }\end{array}$ \\
\hline $\begin{array}{l}\text { Low } \mathrm{CV} \\
\text { risk }\end{array}$ & $\begin{array}{l}\text { Low CV risk NSAID } \\
\text { (naproxen) }\end{array}$ & $\begin{array}{l}\text { NSAID with least GI/ } \\
\text { CV risk (ibuprofen) }\end{array}$ & $\begin{array}{l}\text { 1. NSAID with least GI/CV risk plus } \\
\text { PPI/H2RA }{ }^{\mathrm{a}} \text { (ibuprofen/naproxen) } \\
\text { 2. Celecoxib }\end{array}$ & $\begin{array}{l}\text { 1. Celecoxib }+ \text { PPI/H2RA } \\
\text { 2. NSAID with least GI risk plus } \\
\text { PPI/H2RA }{ }^{\mathrm{a}} \text { (ibuprofen) }\end{array}$ \\
\hline $\begin{array}{l}\text { High CV } \\
\text { risk }\end{array}$ & $\begin{array}{l}\text { Low CV risk NSAID } \\
\left(\text { naproxen }+ \text { LDA }^{\text {b }}\right)\end{array}$ & $\begin{array}{l}\text { Low CV risk NSAID } \\
\left(\text { naproxen }+ \text { LDA }^{\text {b }}\right)\end{array}$ & $\begin{array}{l}\text { NSAID with least CV/GI risk + PPI/ } \\
\text { H2RA (naproxen }{ }^{\mathrm{a}}+\text { LDA }^{\mathrm{b}} \text { ) }\end{array}$ & $\begin{array}{l}\text { Avoid NSAIDs if possible, } \\
\text { LDA + PPI }\end{array}$ \\
\hline
\end{tabular}

Adapted from references [18, 19]

$C V$ cardiovascular, GI gastrointestinal, $H 2 R A$ histamine $\mathrm{H}_{2}$ receptor antagonist, LDA low-dose aspirin, NSAID non-steroidal anti-inflammatory drug, PPI proton pump inhibitor

${ }^{a}$ When high doses are needed, fixed-dose combination products of ibuprofen/famotidine or naproxen/esomeprazole might be warranted for adherence benefits

${ }^{\mathrm{b}}$ Aspirin should be administered at least $2 \mathrm{~h}$ before NSAID when indicated 


\section{Conclusion}

Clinicians should continue to use their country of origin's current prescribing information and individual patient assessment to guide their decision making regarding balancing the GI and CV risk of these agents. NSAIDs have clearly improved the quality of life of patients, as demonstrated by the high usage and acceptability of these agents, as well as their documented efficacy in clinical trials. However, this efficacy comes with the potential price of increased CV and GI side effects, especially at the highest and most efficacious dosages. With the information available today, there is insufficient evidence to conclude from a population perspective that there are differences between the major marketed NSAIDs in regard to their potential for $\mathrm{CV}$ events. Though it does appear that the data suggest a difference between naproxen and other NSAIDs, it does not reach the level of substantial supportive evidence. Perhaps, the ongoing PRECISION trial will, when completed, provide further evidence to reach better informed conclusions.

Funding and conflicts of interest Dr. Bello was a previous employee of Searle/Pfizer and has received consulting and speaking fees in the past from Horizon Pharma, ABBVIE, Questcor, and UCB. Dr. Holt was a previous employee of Searle/Pfizer and is currently receiving, or previously has received, consulting fees, directly or indirectly, from Horizon Pharma, Cadence Pharma, Hospira, and Pozen. No funding was received to assist in the preparation of this review article.

Open Access This article is distributed under the terms of the Creative Commons Attribution Noncommercial License which permits any noncommercial use, distribution, and reproduction in any medium, provided the original author(s) and the source are credited.

\section{References}

1. US Food and Drug Administration. FDA briefing document on nonsteroidal anti-inflammatory drugs and cardiovascular thrombotic risk. Joint Meeting of the Arthritis Advisory Committee and the Drug Safety and Risk Management, Advisory Committee, February 10-11, 2014. http://www.fda.gov/ downloads/AdvisoryCommittees/CommitteesMeetingMaterials/ Drugs/ArthritisAdvisoryCommittee/UCM383180.pdf. Accessed February 14, 2014.

2. Saag K, van der Heijde D, Fisher $C$, et al. Rofecoxib, a new cyclooxygenase 2 inhibitor, shows sustained efficacy, comparable with other nonsteroidal anti-inflammatory drugs. Arch Fam Med. 2000;9:1124-34.

3. Day R, Morrison B, Luza A, et al. A randomized trial of the efficacy and tolerability of the coxib rofecoxib vs ibuprofen in patients with osteoarthritis. Arch Intern Med. 2000;160:1781-7.
4. Bensen WG, Fiechtner JJ, McMillen JI, et al. Treatment of osteoarthritis with celecoxib, a cyclooxygenase-2 inhibitor: a randomized controlled trial. Mayo Clin Proc. 1999;74(11): 1095-105.

5. Celebrex ${ }^{\circledR}$ prescribing information. Pfizer, Inc; 2011.

6. Silverstein FE, Faich G, Goldstein JL, et al. Gastrointestinal toxicity with celecoxib vs nonsteroidal anti-inflammatory drugs for osteoarthritis and rheumatoid arthritis the CLASS study: a randomized controlled trial. JAMA. 2000;284:1247-55.

7. Bombardier C, Laine L, Reicin A, et al. Comparison of upper gastrointestinal toxicity of rofecoxib and naproxen in patients with rheumatoid arthritis. N Engl J Med. 2000;343:1520-8.

8. Curfman GD, Morrissey S, Drazen JM. Expression of concern: Bombardier et al., "Comparison of upper gastrointestinal toxicity of rofecoxib and naproxen in patients with rheumatoid arthritis", N Engl J Med 2000;343:1520-1528. N Engl J Med. 2005;353: 2813-4.

9. Curfman GD, Morrissey S, Drazen JM. Expression of concern reaffirmed. N Engl J Med. 2006;354:1193.

10. Bresalier RS, Sandler RS, Quan H, et al. Cardiovascular events associated with rofecoxib in a colorectal adenoma chemoprevention trial. N Engl J Med. 2005;352(11):1092-102.

11. Baron JA, Sandler RS, Bresalier RS, et al. Cardiovascular events associated with rofecoxib: final analysis of the APPROVe trial. Lancet. 2008;372(9651):1756-64.

12. Becker MC, Wang TH, Wisniewski L, et al. Rationale, design, and governance of prospective randomized evaluation of celecoxib integrated safety versus ibuprofen or naproxen (PRECISION), a cardiovascular end point trial of nonsteroidal antiinflammatory agents in patients with arthritis. Am Heart J. 2009;157:606-12.

13. Schjerning Olsen A, Fosbøl EL, Lindhardsen J, et al. Duration of treatment with nonsteroidal anti-inflammatory drugs and impact on risk of death and recurrent myocardial infarction in patients with prior myocardial infarction: a nationwide cohort study. Circulation. 2011;123:2226-35.

14. Coxib and traditional NSAID Trialists' (CNT) Collaboration. Vascular and upper gastrointestinal effects of non-steroidal antiinflammatory drugs: meta-analyses of individual participant data from randomised trials. Lancet. 2013;382(9894):769-79. doi:10. 1016/S0140-6736(13)60900-9 (Epub 2013 May 30).

15. European Medicines Agency, Press Release 06/28/2013, New safety advice for diclofenac-CMDh endorses PRAC recommendation. http://www.ema.europa.eu/ema/index.jsp?curl=pages/ news_and_events/news/2013/06/news_detail_001830.jsp\&mid= WC0b01ac058004d5c1. Accessed 21 April 2014.

16. Altman DG, Bland JM. Statistics notes. Interaction revisited: the difference between two estimates. BMJ. 2003;326:219.

17. Olsen A, Fosbøl EL, Gislason GH. The impact of NSAID treatment on cardiovascular risk-insight from Danish observational data. Basic Clin Pharmacol Toxicol. 2014. doi:10.1111/bcpt. 12244.

18. Lanza FL, Chan FK, Quigley EM. Guidelines for prevention of NSAID-related ulcer complications. Am J Gastroenterol. 2009;104(3):728-38.

19. PL detail-document, managing NSAID risks. Pharmacist's Letter/ Prescriber's Letter, July 2013. 